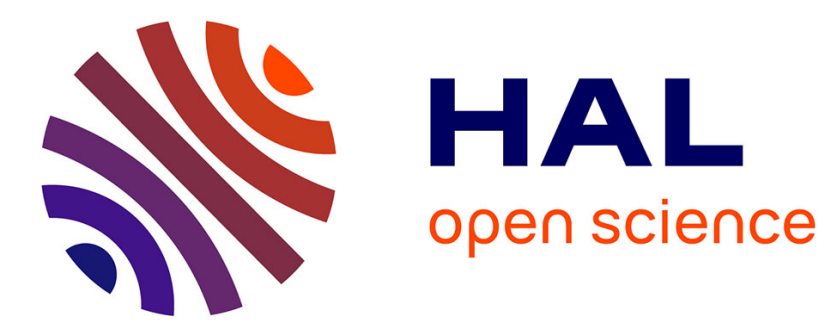

\title{
Modeling very oscillating signals. Application to image processing
}

Gilles Aubert, Jean-François Aujol

\section{To cite this version:}

Gilles Aubert, Jean-François Aujol. Modeling very oscillating signals. Application to image processing. RR-4878, INRIA. 2003. inria-00071705

\section{HAL Id: inria-00071705 https://hal.inria.fr/inria-00071705}

Submitted on 23 May 2006

HAL is a multi-disciplinary open access archive for the deposit and dissemination of scientific research documents, whether they are published or not. The documents may come from teaching and research institutions in France or abroad, or from public or private research centers.
L'archive ouverte pluridisciplinaire $\mathbf{H A L}$, est destinée au dépôt et à la diffusion de documents scientifiques de niveau recherche, publiés ou non, émanant des établissements d'enseignement et de recherche français ou étrangers, des laboratoires publics ou privés. 
INSTITUT NATIONAL DE RECHERCHE EN INFORMATIQUE ET EN AUTOMATIQUE

\section{Modeling very oscillating signals. Application to image processing.}

Gilles Aubert — Jean-François Aujol

$\mathbf{N}^{\circ} 4878$

July 2003

THÈME 3 



\title{
Modeling very oscillating signals. Application to image processing.
}

\author{
Gilles Aubert* ${ }^{*}$, Jean-François Aujol ${ }^{\dagger}$ \\ Thème 3 - Interaction homme-machine, \\ images, données, connaissances \\ Projet Ariana \\ Rapport de recherche $n^{\circ} 4878$ - July 2003 - 30 pages
}

\begin{abstract}
This article is a companion paper of a previous work [5] where we have developed the numerical analysis of a variational model first introduced by L. Rudin, S. Osher and E. Fatemi [23] and revisited by Y. Meyer [18] for removing the noise and capturing textures in an image. The basic idea in this model is to decompose $f$ into two components $(u+v)$ and then to search for $(u, v)$ as a minimizer of an energy functional. The first component $u$ belongs to $B V$ and contains geometrical informations while the second one $v$ is sought in a space $G$ which contains signals with large oscillations, i.e. noise and textures. In [18] Y. Meyer carried out his study in the whole $\mathbb{R}^{2}$ and his approach is rather built on harmonic analysis tools. We place ourselves in the case of a bounded set $\Omega$ of $\mathbb{R}^{2}$ which is the proper setting for image processing and our approach is based upon functional analysis arguments. We define in this context the space $G$, give some of its properties and then study in this continuous setting the energy functional which allows us to recover the components $u$ and $v$.
\end{abstract}

Key-words: Sobolev spaces, functions with bounded variations, PDEs, oscillating patterns, image decomposition, convex analysis, optimization, calculus of variations.

\footnotetext{
* Laboratoire J. A. Dieudonné, UMR CNRS 6621, Université de Nice-Sophia-Antipolis, Parc Valrose, 06108 Nice Cedex 2, France

$\dagger$ également membre du laboratoire J.A Dieudonné, Université de Nice-Sophia-Antipolis
} 


\section{Modélisation de signaux fortement oscillants. Application en traitement d'images.}

Résumé : Cet article complète le travail présenté dans [5] dans lequel nous avions développé l'analyse numérique d'un modéle variationel, initialement introduit par L. Rudin, S. Osher and E. Fatemi [23], et revisité depuis par Y. Meyer [18], pour supprimer le bruit et isoler les textures dans une image. Dans un tel modèle, on décompose l'image $f$ en deux composantes $(u+v), u$ et $v$ minimisant une énergie. La première composante $u$ appartient à $B V$ et contient l'information géométrique de l'image, alors que la seconde $v$ appartient à un espace $G$ qui contient les signaux à fortes oscillations, i.e. le bruit et les textures. Dans [18], Y. Meyer effectue son étude dans $\mathbb{R}^{2}$ entier, et son approche repose principalement sur des outils d'analyse harmonique. Nous nous plaçons dans le cas d'un ouvert borné $\Omega$ de $\mathbb{R}^{2}$, ce qui constitue le cadre adapté au traitement d'images, et notre approche repose sur des arguments d'analyse fonctionelle. Nous définissons l'espace $G$ dans ce cadre puis donnons quelques unes de ses propriétés. Nous étudions ensuite la fonctionelle permettant de calculer les composantes $u$ et $v$.

Mots-clés : Espaces de Sobolev, Fonctions à variations bornées, EDP, signaux oscillants, décomposition d'images, analyse convexe, optimisation, calcul des variations. 


\section{Contents}

.1 Introduction $\quad 4$

1.1 Rudin-Osher-Fatemi model .................... 4

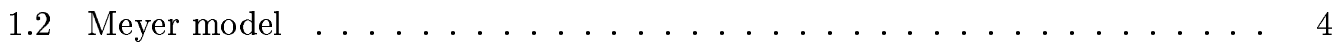

1.3 Vese-Osher model . . . . . . . . . . . . . . . . . . 5

1.4 Aujol-Aubert-Blanc-Féraud-Chambolle model . . . . . . . . . . . . . 5

.2 A space for modeling oscillating patterns $\quad 6$

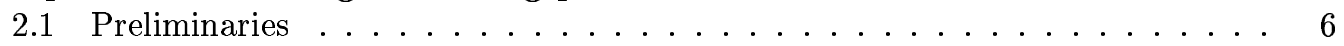

2.2 Definition and properties of $G(\Omega) \ldots \ldots \ldots \ldots$

$\begin{array}{lr}3 \text { Study of Meyer problem } & 14\end{array}$

$\begin{array}{lr}.4 \text { An algorithm to solve Meyer problem } & 17\end{array}$

4.1 Total variation minimization as a projection . . . . . . . . . . . . 17

4.2 Solving Meyer problem as a projection . . . . . . . . . . . . . 18

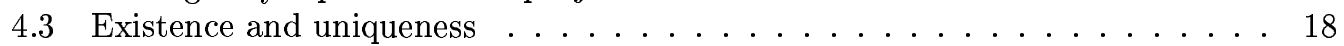

4.4 Characterization of the minimizer $(\hat{u}, \hat{v}) \ldots \ldots . \ldots 20$

4.5 Study of the limit problem as $\lambda \rightarrow 0 \ldots \ldots \ldots$

4.6 Limit when $\lambda \rightarrow 0$...................... . . . 24

4.7 Numerical results . . . . . . . . . . . . . . . . . 26 


\section{Introduction}

\subsection{Rudin-Osher-Fatemi model}

Denoising an image is one of the major goal of image processing [4]. There have been many attempts to do this. One of the most effective method is the total variation minimization as proposed in [23]. This model relies on the assumption that $B V(\Omega)$, the space of functions with bounded variation, is a good space to study images (even if it is known that such an assumption is too restrictive [2]). In [23], the authors decompose an image $f$ into a component $u$ belonging to $B V(\Omega)$ and a component $v$ in $L^{2}(\Omega)$. In this model $v$ is supposed to be the noise. In such an approach, they minimize:

$$
\inf _{(u, v) \in B V(\Omega) \times L^{2}(\Omega) / f=u+v}\left(\int|D u|+\frac{1}{2 \lambda}\|v\|_{L^{2}(\Omega)}\right)
$$

where $\int|D u|$ stands for the total variation of $u$. In practice, they try to compute a numerical solution of the Euler-Lagrange equation associated to (1.1). The mathematical study of (1.1) has been done in [10].

In [18], Y. Meyer shows that the model in [23] does not reach its aim. In particular, if $f$ is the characteristic function of a bounded domain with a $C^{\infty}$-boundary, then $f$ is not preserved by the Rudin-Osher-Fatemi model (contrary to what should be expected).

\section{$1.2 \quad$ Meyer model}

In [18], Y. Meyer suggests a new decomposition. He proposes the following model:

$$
\inf _{(u, v) \in B V\left(\mathbb{R}^{2}\right) \times G\left(\mathbb{R}^{2}\right) / f=u+v}\left(\int|D u|+\lambda\|v\|_{G\left(\mathbb{R}^{2}\right)}\right)
$$

where the Banach space $G\left(\mathbb{R}^{2}\right)$ contains signals with large oscillations, and thus in particular textures and noise. We give here the definition of $G\left(\mathbb{R}^{2}\right)$.

Definition 1.1. $G\left(\mathbb{R}^{2}\right)$ is the Banach space composed of distributions $f$ which can be written

$$
f=\partial_{1} g_{1}+\partial_{2} g_{2}=\operatorname{div}(g)
$$

with $g_{1}$ and $g_{2}$ in $L^{\infty}\left(\mathbb{R}^{2}\right)$. The space $G\left(\mathbb{R}^{2}\right)$ is endowed with the following norm:

$$
\begin{array}{r}
\|v\|_{G\left(\mathbb{R}^{2}\right)}=\inf \left\{\|g\|_{L^{\infty}\left(\mathbb{R}^{2}\right)}=\underset{x \in \mathbb{R}^{2}}{\operatorname{ess} \sup _{x}|g(x)| / v=\operatorname{div}(g), g=\left(g_{1}, g_{2}\right),}\right. \\
\left.g_{1} \in L^{\infty}\left(\mathbb{R}^{2}\right), g_{2} \in L^{\infty}\left(\mathbb{R}^{2}\right),|g(x)|=\sqrt{\left(\left|g_{1}\right|^{2}+\left|g_{2}\right|^{2}\right)(x)}\right\}
\end{array}
$$

INRIA 
$B V\left(\mathbb{R}^{2}\right)$ has no simple dual space (see [3]). However, as shown by Y. Meyer [18], $G\left(\mathbb{R}^{2}\right)$ is the dual space of the closure in $B V\left(\mathbb{R}^{2}\right)$ of the Schwartz class. So it is very related to the dual space of $B V\left(\mathbb{R}^{2}\right)$. This is a motivation to decompose a function $f$ on $B V\left(\mathbb{R}^{2}\right)+G\left(\mathbb{R}^{2}\right)$. This is also why the divergence operator naturally appears in the definition of $G\left(\mathbb{R}^{2}\right)$, since the gradient and the divergence operators are dual operators.

A function belonging to $G$ may have large oscillations and nevertheless have a small norm. Thus the norm on $G$ is well-adapted to capture the oscillations of a function.

In [18], the author works on the whole $\mathbb{R}^{2}$. This is not the proper setting for image processing (it skips the problem of boundary conditions). We intend here to give a definition of $G$ for a bounded domain $\Omega \subset \mathbb{R}^{2}$. Moreover, [18] gives no way to compute numerically a solution of (1.2). To fill this gap, some numerical models have been proposed in the literature $[25,21,5]$. We review below some of them.

\subsection{Vese-Osher model}

L. Vese and S. Osher were the first authors to numerically tackle Meyer program [25]. They actually solve the problem:

$$
\inf _{(u, v) \in B V(\Omega) \times G(\Omega)}\left(\int|D u|+\lambda\|f-u-v\|_{2}^{2}+\mu\|v\|_{G(\Omega)}\right)
$$

where $\Omega$ is a bounded open set. To compute their solution, they replace the term $\|v\|_{G(\Omega)}$ by $\left\|\sqrt{g_{1}^{2}+g_{2}^{2}}\right\|_{p}$ (where $v=\operatorname{div}\left(g_{1}, g_{2}\right)$ ). Then they formally derive the Euler-Lagrange equations from (1.5). For numerical reasons, the authors use the value $p=1$ (they claim they made experiments for $p=1 \ldots 10$, and that they did not see any visual difference). They report good numerical results. See also [21] for another related model concerning the case $\lambda=+\infty$ and $p=2$.

\subsection{Aujol-Aubert-Blanc-Féraud-Chambolle model}

Inspired from the work by A. Chambolle [9], the authors of [5] propose a relevant approach to solve Meyer problem. They consider the following functional defined on $L^{2}(\Omega) \times L^{2}(\Omega)$ :

$$
F_{\lambda, \mu}(u, v)= \begin{cases}\int_{\Omega}|D u|+\frac{1}{2 \lambda}\|f-u-v\|_{L^{2}(\Omega)}^{2} & \text { if }(u, v) \in B V(\Omega) \times G_{\mu}(\Omega) \\ +\infty & \text { otherwise }\end{cases}
$$

where $G_{\mu}(\Omega)=\left\{v \in G(\Omega) /\|v\|_{G(\Omega)} \leq \mu\right\}$. And the problem to solve is:

$$
\inf _{L^{2}(\Omega) \times L^{2}(\Omega)} F_{\lambda, \mu}(u, v)
$$

The authors of [5] present their model in a discrete framework. They carry out a complete mathematical analysis of their discrete model, showing how it approximately solves Meyer problem. 
The aim of this paper is to carry out the mathematical analysis of (1.2) and (1.7) in a continuous setting. This will clarify a few facts about Meyer ideas developed in [18]. We first need to give the proper definition of $G(\Omega)$ when $\Omega$ is a bounded domain of $\mathbb{R}^{2}$. This will be done in Section 2, where we fix some notations, define $G(\Omega)$, and then give some properties, explaining in particular why this space is so interesting to model signals with strong oscillations. Thanks to these preliminaries, we solve Meyer problem in Section 3, showing the existence of a solution for (1.2). In Section 4, we carry out the mathematical analysis of (1.7) in the continuous framework we have introduced. This proves the relevance of the numerical algorithm developed in [5]. We end this paper by showing some numerical results on images.

\section{A space for modeling oscillating patterns}

\subsection{Preliminaries}

Throughout our study, we will use the following classical distributional spaces. $\Omega \subset R^{N}$ will denote an open bounded set of $R^{N}$.

- $\mathcal{D}(\Omega)=C_{c}^{\infty}(\Omega)$ is the set of functions in $C^{\infty}(\Omega)$ with compact support in $\Omega$. We denote by $\mathcal{D}^{\prime}(\Omega)$ the dual space of $\mathcal{D}(\Omega)$, i.e. the space of distributions on $\Omega . \mathcal{D}(\bar{\Omega})$ is the set of restriction to $\Omega$ of functions in $\mathcal{D}\left(\mathbb{R}^{N}\right)=C_{c}^{\infty}\left(\mathbb{R}^{N}\right)$.

- $W^{m, p}(\Omega)$ denotes the space of functions in $L^{p}(\Omega)$ whose distributional derivatives $D^{\alpha} u$ are in $L^{p}(\Omega), p \in[1,+\infty), m \geq 1, m \in \mathbb{N},|\alpha| \leq m$. $W_{0}^{m, p}(\Omega)$ denotes the space of functions in $W^{m, p}(\Omega)$ with compact support in $\Omega$. For further details on these spaces, we refer the reader to $[1,8,12]$.

- $B V(\Omega)$ is the subspace of functions $u \in L^{1}(\Omega)$ such that the following quantity is finite:

$$
J(u)=\sup \left\{\int_{\Omega} u(x) \operatorname{div}(\xi(x)) d x / \xi \in C_{c}^{\infty}\left(\Omega, \mathbb{R}^{N}\right),\|\xi\|_{L^{\infty}\left(\Omega, \mathbb{R}^{N}\right)} \leq 1\right\}
$$

$B V(\Omega)$ endowed with the norm $\|u\|_{B V}=\|u\|_{L^{1}}+J(u)$ is a Banach space. If $u \in$ $B V(\Omega)$, the distributional derivative $D u$ is a bounded Radon measure and (2.1) corresponds to the total variation $|D u|(\Omega)$.

For $\Omega \subset R^{2}$, we have [3]:

$$
B V(\Omega) \subset L^{2}(\Omega)
$$

For further details on $B V(\Omega)$, we refer the reader to $[3,13]$.

We will need the notion of regular set. 
Definition 2.1. We say that $\Omega \subset \mathbb{R}^{2}$ is regular if it verifies the following property: there exist $\eta>0$ and $A>0$ depending only on $\Omega$ such that for all $\epsilon \in(0, \eta)$, and for all $x \in \Omega$,

$$
|B(x, \epsilon) \bigcap \Omega| \geq A \epsilon^{2}
$$

where $B(x, \epsilon)=\left\{y \in \mathbb{R}^{2} /\|x-y\| \leq \epsilon\right\}$, and $|B(x, \epsilon) \bigcap \Omega|$ denotes the Lebesgue measure of $B(x, \epsilon) \cap \Omega$.

Remark: If $\Omega$ is such that $\partial \Omega$ is $C^{2}$ and of finite length, then $\Omega$ is regular. The same is true if $\Omega$ is a polygonal set.

We will also need the following result:

Lemma 2.1. $\Omega$ is assumed to be regular. Let us consider a sequence $w_{n}: \Omega \rightarrow \mathbb{R}$ such that:

1. $\left\|w_{n}\right\|_{L^{1}(\Omega)} \rightarrow 0$ when $n \rightarrow+\infty$.

2. There exists $\theta \in(0,1)$ and $C>0$ such that for all $x$ and $y \in \Omega,\left|w_{n}(x)-w_{n}(y)\right| \leq$ $C|x-y|^{\theta}$.

Then $\left\|w_{n}\right\|_{L^{\infty}(\Omega)} \rightarrow 0$ when $n \rightarrow+\infty$.

Proof: Let us fix $x \in \Omega$. We can write $w_{n}(x)=w_{n}(x)-w_{n}(y)+w_{n}(y)$. This implies $\left|w_{n}(x)\right| \leq\left|w_{n}(x)-w_{n}(y)\right|+\left|w_{n}(y)\right|$. Using assumption 2., we get:

$$
\left|w_{n}(x)\right| \leq C|x-y|^{\theta}+\left|w_{n}(y)\right|
$$

Let $\epsilon \in(0, \eta)$ ( $\eta$ as in Definition 2.1), and let us denote by $B=B(x, \epsilon)=\left\{y \in \mathbb{R}^{2} /|x-y| \leq\right.$ $\epsilon\}$. From (2.4), we have

$$
|B \bigcap \Omega|\left|w_{n}(x)\right| \leq C \int_{B \cap \Omega}|x-y|^{\theta} d y+\int_{B \cap \Omega}\left|w_{n}(y)\right| d y
$$

Hence

$$
|B \bigcap \Omega|\left|w_{n}(x)\right| \leq C \int_{B}|x-y|^{\theta} d y+\int_{\Omega}\left|w_{n}(y)\right| d y
$$

Since $\Omega$ is regular, we have $|B \bigcap \Omega| \geq A \epsilon^{2}$. And using polar coordinates, we get:

$$
\int_{B}|x-y|^{\theta} d y=2 \pi \int_{0}^{\epsilon} r^{\theta} r d r=\frac{2 \pi}{\theta+2} \epsilon^{\theta+2}
$$

From (2.6), we obtain:

$$
A \epsilon^{2}\left|w_{n}(x)\right| \leq \frac{2 \pi C}{\theta+2} \epsilon^{\theta+2}+\int_{\Omega}\left|w_{n}(y)\right| d y
$$


i.e:

$$
\left|w_{n}(x)\right| \leq \frac{2 \pi C}{A(\theta+2)} \epsilon^{\theta}+\frac{1}{A \epsilon^{2}} \int_{\Omega}\left|w_{n}(y)\right| d y
$$

Denoting by $C^{\prime}=\frac{2 \pi C}{A(\theta+2)}$, we get from (2.9) that:

$$
\left|w_{n}(x)\right| \leq C^{\prime} \epsilon^{\theta}+\frac{1}{A \epsilon^{2}} \int_{\Omega}\left|w_{n}(y)\right| d y
$$

Hence:

$$
\left\|w_{n}\right\|_{L^{\infty}(\Omega)} \leq C^{\prime} \epsilon^{\theta}+\frac{1}{A \epsilon^{2}}\left\|w_{n}\right\|_{L^{1}(\Omega)}
$$

Letting $n \rightarrow+\infty$, we get (since $\left\|w_{n}\right\|_{L^{1}(\Omega)} \rightarrow 0$ ):

$$
\lim _{n \rightarrow+\infty}\left\|w_{n}\right\|_{L^{\infty}(\Omega)} \leq C^{\prime} \epsilon^{\theta}
$$

As (2.12) holds for all $\epsilon \in(0, \eta)$, we can let $\epsilon \rightarrow 0^{+}$, and we obtain that $\left\|w_{n}\right\|_{L^{\infty}(\Omega)} \rightarrow 0$ when $n \rightarrow+\infty$.

\subsection{Definition and properties of $G(\Omega)$}

In all the sequel, we denote by $\Omega$ a bounded connected open set of $\mathbb{R}^{2}$ with a Lipschitz boundary. We adapt Definition 1.1 concerning the space $G$ to the case of $\Omega$. We are going to consider a subspace of the Banach space $W^{-1, \infty}(\Omega)=\left(W_{0}^{1,1}(\Omega)\right)^{\prime}$ (the dual space of $\left.W_{0}^{1,1}(\Omega)\right)$.

Definition 2.2. $G(\Omega)$ is the subspace of $W^{-1, \infty}(\Omega)$ defined by:

$$
G(\Omega)=\left\{v \in L^{2}(\Omega) / v=\operatorname{div} \xi, \xi \in L^{\infty}\left(\Omega, \mathbb{R}^{2}\right), \xi . N=0 \text { on } \partial \Omega\right\}
$$

On $G(\Omega)$, the following norm is defined:

$$
\|v\|_{G(\Omega)}=\inf \left\{\|\xi\|_{L^{\infty}\left(\Omega, \mathbb{R}^{2}\right)} / v=\operatorname{div} \xi, \xi \cdot N=0 \text { on } \partial \Omega\right\}
$$

Remark: In Definition 2.2, since $\operatorname{div} \xi \in L^{2}(\Omega)$ and $\xi \in L^{\infty}\left(\Omega, \mathbb{R}^{2}\right)$, we can define $\xi . N$ on $\partial \Omega$ (see [17]).

We have the following simple characterization of $G(\Omega)$ :

\section{Proposition 2.1.}

$$
G(\Omega)=\left\{v \in L^{2}(\Omega) / \int_{\Omega} v=0\right\}
$$

INRIA 
Proof: Let us denote by $H(\Omega)$ the right-hand-side of (2.15). We split the proof into two steps.

Step 1:

Let $v$ be in $G(\Omega)$. Then from $(2.13)$ it is immediate that $\int_{\Omega} v=0$, i.e. $v \in H(\Omega)$.

Step 2:

Let $v$ be in $H(\Omega)$. Then from [6] (Theorem 3') (see also [7]), there exists $\xi \in C^{0}\left(\bar{\Omega}, \mathbb{R}^{2}\right) \cap$ $W^{1,2}\left(\Omega, \mathbb{R}^{2}\right)$ such that $v=\operatorname{div} \xi$ and $\xi=0$ on $\partial \Omega$. In particular, we have $\xi \in L^{\infty}\left(\Omega, \mathbb{R}^{2}\right)$ and $\xi . N=0$ on $\partial \Omega$. Thus $v \in G(\Omega)$.

Remark: Let us stress here how powerful the result in $[7,6]$ is. It deals with the limit case $v$ in $L^{q}(\Omega), q=2$, when the dimension of the space is $N=2$. The classical method for tackling the equation $\operatorname{div} \xi=v$ with $\xi . N=0$ on $\partial \Omega$ consists in solving the problem $\Delta u=v$ with $\frac{\partial u}{\partial N}=0$ on $\partial \Omega$, and to set $\xi=\nabla u$. If $v$ is in $L^{q}(\Omega)$ with $q>2$ this last problem admits a unique solution (up to a constant) in $W^{2, q}(\Omega)$. Moreover, thanks to standard Sobolev embeddings (see $[12,8,14]), \xi=\nabla u$ belongs to $L^{\infty}\left(\Omega, \mathbb{R}^{2}\right)$. If $q=2$, the result is not true and the classical approach does not work. So the result by Bourgain and Brezis is very sharp.

We next introduce a family of convex subsets of $G(\Omega)$. These convex sets will be useful for approximating Meyer problem.

Definition 2.3. Let $G_{\mu}(\Omega)$ the family of subset defined by $(\mu>0)$ :

$$
G_{\mu}(\Omega)=\left\{v \in G(\Omega) /\|v\|_{G(\Omega)} \leq \mu\right\}
$$

Lemma 2.2. $G_{\mu}(\Omega)$ is closed for the $L^{2}(\Omega)$-weak topology.

Proof of Lemma 2.2 Let $\left(v_{n}\right)$ be a sequence in $G_{\mu}(\Omega)$ such that there exists $\hat{v} \in L^{2}(\Omega)$ such that $v_{n} \rightarrow \hat{v}$ in $L^{2}(\Omega)$-weak. We have $v_{n}=\operatorname{div} \xi_{n}$, with $\xi_{n}$ such that $\left\|\xi_{n}\right\|_{L^{\infty}\left(\Omega, \mathbb{R}^{2}\right)} \leq \mu$ and $\xi_{n} \cdot N=0$ on $\partial \Omega$.

As $\left\|\xi_{n}\right\|_{L^{\infty}\left(\Omega, \mathbb{R}^{2}\right)} \leq \mu$, there exists $\hat{\xi} \in L^{\infty}\left(\Omega, \mathbb{R}^{2}\right)$ such that, up to an extraction:

$$
\xi_{n} \rightarrow \hat{\xi} \text { in } L^{\infty}\left(\Omega, \mathbb{R}^{2}\right) \text { weak * }
$$

and $\|\hat{\xi}\|_{L^{\infty}\left(\Omega, \mathbb{R}^{2}\right)} \leq \mu$. Moreover if $\phi \in C_{c}^{\infty}(\Omega)$ :

$$
\int_{\Omega} v_{n} \phi d x=\int_{\Omega} \operatorname{div} \xi_{n} \phi d x=-\int_{\Omega} \xi_{n} \nabla \phi d x
$$

Thus as $n \rightarrow+\infty$, we get

$$
\int_{\Omega} \hat{v} \phi d x=-\int_{\Omega} \hat{\xi} \nabla \phi d x=\int_{\Omega} \operatorname{div} \hat{\xi} \phi d x
$$


Hence $\hat{v}=\operatorname{div} \hat{\xi}$ in $\mathcal{D}^{\prime}(\Omega)$, and since $\hat{v} \in L^{2}(\Omega)$, the equality is true in $L^{2}(\Omega)$.

Now, if $\phi \in \mathcal{D}(\bar{\Omega})$ :

$$
\int_{\Omega} v_{n} \phi d x=\int_{\Omega} \operatorname{div} \xi_{n} \phi d x=-\int_{\Omega} \xi_{n} \nabla \phi d x+\int_{\partial \Omega} \xi_{n} \cdot N \phi
$$

and as $n \rightarrow+\infty$ :

$$
\int_{\Omega} \hat{v} \phi d x=-\int_{\Omega} \hat{\xi} \nabla \phi d x+\lim _{n \rightarrow+\infty} \int_{\partial \Omega} \xi_{n} \cdot N \phi
$$

i.e:

$$
\int_{\Omega} \hat{v} \phi d x=\int_{\Omega} \operatorname{div} \hat{\xi} \phi d x-\int_{\partial \Omega} \xi \cdot N \phi+\lim _{n \rightarrow+\infty} \int_{\partial \Omega} \xi_{n} \cdot N \phi
$$

As $\hat{v}=\operatorname{div} \hat{\xi}$, we get:

$$
0=-\int_{\partial \Omega} \hat{\xi} \cdot N \phi+\lim _{n \rightarrow+\infty} \int_{\partial \Omega} \xi_{n} \cdot N \phi
$$

And as $\xi_{n} \cdot N=0$ on $\partial \Omega$, we obtain:

$$
0=\int_{\partial \Omega} \xi \cdot N \phi \text { for all } \phi \in \mathcal{D}(\bar{\Omega})
$$

Hence $\hat{\xi} \cdot N=0$ on $\partial \Omega$ (in $H^{-1 / 2}(\partial \Omega)$ ) (see [17]).

\section{Consequences:}

1. Since $G_{\mu}(\Omega)$ is convex, we deduce from Lemma 2.2 that $G_{\mu}(\Omega)$ is also closed for the $L^{2}(\Omega)$-strong topology.

2. The characteristic function of $G_{\mu}(\Omega)$ is lsc (lower-semicontinuous) for both the strong and the weak topologies of $L^{2}(\Omega)$ (see [8]).

\section{Remarks:}

1. Let us denote by $K(\Omega)$ the closure in $L^{2}(\Omega)$ of the set:

$$
\left\{\operatorname{div} \xi, \xi \in C_{c}^{\infty}\left(\Omega, \mathbb{R}^{2}\right),\|\xi\|_{L^{\infty}\left(\Omega, \mathbb{R}^{2}\right)} \leq 1\right\}
$$

Using Lemma 2.2 and some results in [24], one can prove that $K(\Omega)=G_{1}(\Omega)$.

2. Let us consider the closure in $L^{2}(\Omega)$ of the set:

$$
\left\{\operatorname{div} \xi, \xi \in C_{c}^{\infty}\left(\Omega, \mathbb{R}^{2}\right)\right\}
$$

Using Lemma 2.2 and some results in [24] and [7], one can prove that this set coincides with $G(\Omega)$. 
We also have the following result:

Lemma 2.3. If $v \in G(\Omega)$, then there exists $\xi \in L^{\infty}\left(\Omega, \mathbb{R}^{2}\right)$ with $v=\operatorname{div} \xi$ and $\xi \cdot N=0$ on $\partial \Omega$, and such that $\|v\|_{G}=\|\xi\|_{L^{\infty}\left(\Omega, \mathbb{R}^{2}\right)}$.

Proof: Let $v \in G(\Omega)$. Let us consider a sequence $\xi_{n} \in L^{\infty}\left(\Omega, \mathbb{R}^{2}\right)$ with $v=\operatorname{div} \xi_{n}$ and $\xi_{n} . N=0$ on $\partial \Omega$, and such that $\left\|\xi_{n}\right\|_{L^{\infty}(\Omega)} \rightarrow\|v\|_{G}$. There exists $\xi \in L^{\infty}\left(\Omega, \mathbb{R}^{2}\right)$ such that, up to an extraction,

$$
\xi_{n} \rightarrow \xi \text { in } L^{\infty}\left(\Omega, \mathbb{R}^{2}\right) \text { weak * }
$$

Then, as in the proof of Lemma 2.2, we can show that $\xi . N=0$ on $\partial \Omega$ and that $v=\operatorname{div} \xi$.

Main property: The following lemma is due to Y. Meyer [18]. But it was stated in the case of $\Omega=\mathbb{R}^{2}$, and the proof relied upon harmonic analysis tools. Thanks to our definition of $G(\Omega)$, we can formulate it in the case when $\Omega$ is bounded. Our proof relies upon functional analysis arguments.

Lemma 2.4. let $f_{n}, n \geq 1$ be a sequence of functions in $L^{q}(\Omega) \bigcap G(\Omega)$ with the two following properties ( $\Omega$ is regular with a lipschitz boundary):

1. There exists $q>2$ and $C>0$ such that $\left\|f_{n}\right\|_{L^{q}(\Omega)} \leq C$.

2. The sequence $f_{n}$ converges to 0 in the distributional sense (i.e. in $\mathcal{D}^{\prime}(\Omega)$ ).

Then $\left\|f_{n}\right\|_{G}$ converges to 0 when $n$ goes to infinity.

This result explains why the norm in $G(\Omega)$ is a good norm to tackle signals with strong oscillations. It will be easier with this norm to capture such signals in a minimization process than with a classical $L^{2}$-norm.

Proof of Lemma 2.4: We split the proof into two steps:

Step 1:

Let us consider a sequence $f_{n} \in L^{q}(\Omega) \bigcap G(\Omega)$ satisfying assumption 1. and let us define the Neumann problem:

$$
\left\{\begin{array}{l}
\Delta u_{n}=f_{n} \text { in } \Omega \\
\frac{\partial u_{n}}{\partial N}=0 \text { on } \partial \Omega
\end{array}\right.
$$

We recall that as $f_{n} \in G(\Omega)$, we also have $\int_{\Omega} f_{n} d x=0$. We know (see $[15,20,11]$ ) that problem (2.28) admits a solution $u_{n} \in W^{2, q}(\Omega)$. But since $q>2$ and $\Omega$ bounded, it is standard (see $[8,1])$ that there exists $\theta \in(0,1)$ such that $W^{2, q}(\Omega)$ is continously embedded 
in $C^{1, \theta}$ (thanks to Morrey's theorem). We denote by $g_{n}=\nabla u_{n}$. We thus have $g_{n} \in C^{0, \theta}\left(\mathbb{R}^{2}\right)$. Moreover (see $[8,1]$ ), if $x$ and $y \in \Omega$, we have:

$$
\left|g_{n}(x)-g_{n}(y)\right| \leq A_{1}(\Omega, q, N)\left\|g_{n}\right\|_{W^{1, q}\left(\Omega, \mathbb{R}^{2}\right)}|x-y|^{\theta}
$$

where $A_{1}(\Omega, q, N)$ is a constant which depends only on $\Omega, q$ and the space dimension $N=2$.

Let us consider again problem (2.28). From $[20,19]$, we also know that there exists a constant $B>0$ such that:

$$
\left\|u_{n}\right\|_{W^{2, q}(\Omega)} \leq B\left\|f_{n}\right\|_{L^{q}(\Omega)}
$$

And as we assume that $\left\|f_{n}\right\|_{L^{q}(\Omega)} \leq C$, we get:

$$
\left\|u_{n}\right\|_{W^{2, q}(\Omega)} \leq B . C
$$

But since $g_{n}=\nabla u_{n}$, we have $\left\|g_{n}\right\|_{W^{1, q}(\Omega)} \leq\left\|u_{n}\right\|_{W^{2, q}(\Omega)}$. Thus, from (2.31):

$$
\left\|g_{n}\right\|_{W^{1, q}\left(\Omega, \mathbb{R}^{2}\right)} \leq B . C
$$

Then we deduce from (2.29) and (2.32) that for all $x$ and $y \in \Omega$,we have:

$$
\left|g_{n}(x)-g_{n}(y)\right| \leq A_{2}(\Omega, q, N)|x-y|^{\theta}
$$

where $A_{2}(\Omega, q, N)$ is a constant which depends only on $\Omega, q$ and the space dimension $N=2$.

Step 2:

From (2.31) we deduce that there exists $u \in W^{2, q}(\Omega)$ such that up to an extraction,

$$
u_{n} \rightarrow u \text { in } W^{2, q}(\Omega) \text { weak }
$$

And by the compact embedding of $W^{2, q}(\Omega)$ into $W^{1, q}(\Omega)$ (see $[1,8]$ ):

$$
u_{n} \rightarrow u \text { in } W^{1, q}(\Omega) \text { strong }
$$

Let us now mutliply equation (2.28) by a function $\phi \in \mathcal{D}(\Omega)$ :

$$
\int_{\Omega} \Delta u_{n} \phi d x=\int_{\Omega} f_{n} \phi d x
$$

by integrating by part we get

$$
-\int_{\Omega} \nabla u_{n} \nabla \phi d x=\int_{\Omega} f_{n} \phi d x
$$

and since $f_{n} \rightarrow 0$ in $\mathcal{D}^{\prime}(\Omega)$ as $n \rightarrow+\infty$, we deduce:

$$
\lim _{n \rightarrow+\infty} \int_{\Omega} \nabla u_{n} \nabla \phi d x=0
$$

INRIA 
But $u_{n} \rightarrow u$ in $W^{1, q}(\Omega)$, therefore: $\int_{\Omega} \nabla u \nabla \phi d x=0$, i.e.:

$$
-\int_{\Omega} \Delta u \phi d x=0 \forall \phi \in \mathcal{D}(\Omega)
$$

which implies $\Delta u=0$ in $\mathcal{D}^{\prime}(\Omega)$. Since $u$ is in $W^{2, q}(\Omega)$, we have $\Delta u \in L^{q}(\Omega)$. Therefore $\Delta u=0$ in $L^{q}(\Omega)$.Let us now mutliply equation $(2.28)$ by a function $\phi \in \mathcal{D}(\bar{\Omega})$ :

$$
\int_{\Omega} \Delta u_{n} \phi d x=\int_{\Omega} f_{n} \phi d x
$$

Since $\left\|f_{n}\right\|_{L^{q}(\Omega)} \leq C$, there exists $f \in L^{q}(\Omega)$ such that, up to an extraction, $f_{n} \rightarrow f$ in $L^{q}(\Omega)$ when $n \rightarrow+\infty$. And since $f_{n}$ converges to 0 in the distributional sense, we have $f=0$. Thus $\lim _{n \rightarrow+\infty} \int_{\Omega} \Delta u_{n} \phi d x=0$. By integrating by part we get

$$
\int_{\Omega} \Delta u_{n} \phi d x=\underbrace{\int_{\partial \Omega} \nabla u_{n} \cdot N \phi}_{=0 \text { since } \frac{\partial u_{n}}{\partial N}=0}-\int_{\Omega} \nabla u_{n} \nabla \phi d x
$$

As $n \rightarrow+\infty$, we get (since $u_{n} \rightarrow u$ in $W^{1, q}(\Omega)$ ):

$$
\int_{\Omega} \nabla u \nabla \phi d x=0 \forall \phi \in \mathcal{D}(\bar{\Omega})
$$

As $\Delta u=0$ in $L^{q}(\Omega)$, we also have $\int_{\Omega} \Delta u \phi d x=0$ if $\phi$ in $\mathcal{D}(\bar{\Omega})$. Using Stokes formula once again, we obtain:

$$
\int_{\partial \Omega} \frac{\partial u}{\partial N} \phi=\int_{\Omega} \nabla u \nabla \phi d x+\int_{\Omega} \Delta u \phi d x
$$

We conclude that $\int_{\partial \Omega} \frac{\partial u}{\partial N} \phi=0$ for all $\phi$ in $\mathcal{D}(\bar{\Omega})$. We then deduce (see [24]) that $\frac{\partial u}{\partial N}=0$ on $\partial \Omega$. We therefore have shown that $u \in W^{2, q}(\Omega)$ is the solution of the following problem:

$$
\left\{\begin{array}{c}
\Delta u=0 \text { in } \Omega \\
\frac{\partial u_{n}}{\partial N}=0 \text { on } \partial \Omega
\end{array}\right.
$$

We conclude that $\nabla u=0$ in $L^{2}(\Omega)$ (it suffices to integrate by part $\int_{\Omega} u \Delta u d x$ ). As $u_{n} \rightarrow u$ in $W^{1, q}(\Omega)$ strong, we see that $\nabla u_{n} \rightarrow 0$ as $n \rightarrow+\infty$ in $L^{2}\left(\Omega, \mathbb{R}^{2}\right)$ strong. The previous reasonning being true for any subsequence extracted from $u_{n}$, we conclude that the whole sequence $\nabla u_{n}$ is such that $\nabla u_{n} \rightarrow 0$ as $n \rightarrow+\infty$ in $L^{2}\left(\Omega, \mathbb{R}^{2}\right)$ strong, i.e. $g_{n}=\nabla u_{n} \rightarrow 0$ in $L^{2}\left(\Omega, \mathbb{R}^{2}\right)$ strong. From Lemma 2.1, we get that:

$$
\left\|g_{n}\right\|_{L^{\infty}\left(\Omega, \mathbb{R}^{2}\right)} \rightarrow 0
$$

Since $f_{n}=\operatorname{div} g_{n}$, we easily deduce from (2.45) that $\left\|f_{n}\right\|_{G} \rightarrow 0$. 
Example: We give an example of a sequence of functions $f_{n}$ fullfilling the hypotheses of Lemma 2.4.

Consider the sequence of functions $f_{n}$ defined on $\Omega=(-\pi, \pi)^{2}$ by:

$$
f_{n}(x, y)=\cos (n x)+\cos (n y)=\operatorname{div}\left(\frac{1}{n}(\sin (n x), \sin (n y))\right)
$$

We thus define

$$
\xi_{n}(x, y)=\frac{1}{n}(\sin (n x), \sin (n y))
$$

We have $\xi_{n} \in L^{\infty}\left(\Omega, \mathbb{R}^{2}\right)$. Moreover, one easily sees that $\xi_{n} \cdot N=0$ on $\partial \Omega$ for all $n>0$. Thus $f_{n} \in G(\Omega)$, and we clearly have:

$$
\left\|f_{n}\right\|_{G(\Omega)} \rightarrow 0 \text { when } n \rightarrow+\infty
$$

Now, let $\phi \in \mathcal{D}(\Omega)$. Then, using Stokes formula, we get:

$$
\begin{aligned}
\int_{\Omega} \phi f_{n} d x d y & =\int_{\Omega} \phi \operatorname{div}\left(\xi_{n}\right) d x d y \\
& =-\int_{\Omega} \nabla \phi \cdot \xi_{n} d x d y+\int_{\partial \Omega} \phi \underbrace{\xi_{n} \cdot N}_{=0 \text { on } \partial \Omega}
\end{aligned}
$$

But

$$
\left|\int_{\Omega} \nabla \phi \cdot \xi_{n} d x d y\right| \leq\|\nabla \phi\|_{L^{1}(\Omega)} \underbrace{\left\|\xi_{n}\right\|_{L^{\infty}\left(\Omega, \mathbb{R}^{2}\right)}}_{\rightarrow 0 \text { when } n \rightarrow+\infty}
$$

We conclude that $f_{n} \rightarrow 0$ in $\mathcal{D}^{\prime}(\Omega)$.

We can compute $\left\|f_{n}\right\|_{L^{3}(\Omega)}^{3}$ :

$$
\left\|f_{n}\right\|_{L^{3}(\Omega)}=\left(\frac{104 \pi}{3}\right)^{1 / 3}
$$

This example shows that the result of Lemma 2.4 is not trivial.

\section{Study of Meyer problem}

Thanks to Section 2, we are now in position to carry out the mathematical study of Meyer problem [18].

Let $f \in L^{q}(\Omega)$ (with $q>2$ ). We recall that the considered problem is:

$$
\inf _{(u, v) \in B V(\Omega) \times G(\Omega) / f=u+v}\left(J(u)+\alpha\|v\|_{G(\Omega)}\right)
$$

where $J(u)$ is the total variation $|D u|$ defined by $(2.1)$.

INRIA 


\section{Remarks:}

1. Since $f$ is an image, we know that $f \in L^{\infty}(\Omega)$. Thus it is not restrictive to suppose $q>2$.

2. Since $\Omega$ is bounded, and since $q>2$, we know that $L^{q}(\Omega) \subset L^{2}(\Omega)$.

3. Since $\Omega \subset \mathbb{R}^{2}$, we have (see [3]) that

$$
B V(\Omega) \subset L^{2}(\Omega)
$$

Before considering problem (3.1), we first need to show that we can always decompose a function $f \in L^{q}(\Omega)$ into two components $(u, v) \in B V(\Omega) \times G(\Omega)$.

Lemma 3.1. Let $f \in L^{q}(\Omega)$ (with $q>2$ ). Then there exists $u \in B V(\Omega)$ and $v \in G(\Omega)$ such that $f=u+v$.

Proof: Let us consider the following problem:

$$
\left\{\begin{array}{c}
-\Delta u+u=f \text { in } \Omega \\
\frac{\partial u}{\partial N}=0 \text { on } \partial \Omega
\end{array}\right.
$$

From [15], we know that there exists a unique $u \in W^{2, q}(\Omega)$ solution of (3.3). Let us denote by $\xi=-\nabla u$. From (3.3), we see that $\xi$ is such that $\xi . N=-\nabla u \cdot N=0$. And since $\operatorname{div} \xi=f-u, \operatorname{div} \xi \in L^{2}(\Omega)$. Moreover, since $q>2$ and $\Omega \subset \mathbb{R}^{2}, \xi \in W^{1, q}\left(\Omega, \mathbb{R}^{2}\right)$ which is continuously embedded in $L^{\infty}\left(\Omega, \mathbb{R}^{2}\right)$ (thanks to Morrey's theorem, see [8,1]). We thus conclude that $v=\operatorname{div} \xi$ belongs to $G(\Omega)$.

Since $\Omega$ is bounded, and $q>2$, we have $W^{2, q}(\Omega) \subset W^{1,1}(\Omega)$. But we know that $W^{1,1}(\Omega) \subset B V(\Omega)$. Hence $u \in B V(\Omega)$.

We now show that problem (3.1) admits at least one solution.

Proposition 3.1. Let $f \in L^{q}(\Omega)$ (with $q>2$ ). Then there exists $\hat{u} \in B V(\Omega)$ and $\hat{v} \in G(\Omega)$ such that $f=\hat{u}+\hat{v}$, and:

$$
J(\hat{u})+\alpha\|\hat{v}\|_{G}=\inf _{(u, v) \in B V(\Omega) \times G(\Omega) / f=u+v}\left(J(u)+\alpha\|v\|_{G}\right)
$$

Proof: Let $\left(u_{n}, v_{n}\right)$ be a minimizing sequence for (3.1) (from Lemma 3.1, we know that the infimum in (3.4) is finite). We thus have

$$
J\left(u_{n}\right) \leq C
$$

and

$$
\left\|v_{n}\right\|_{G} \leq C
$$


Since $v_{n} \in G(\Omega)$, we write $v_{n}=\operatorname{div} \xi_{n}$ with $\xi_{n}$ such that $\left\|\xi_{n}\right\|_{L^{\infty}\left(\Omega, \mathbb{R}^{2}\right)}=\left\|v_{n}\right\|_{G}$. We therefore get $\left\|\xi_{n}\right\|_{L^{\infty}\left(\Omega, \mathbb{R}^{2}\right)} \leq C$. Hence there exists $\hat{\xi} \in L^{\infty}\left(\Omega, \mathbb{R}^{2}\right)$ such that (up to a subsequence)

$$
\xi_{n} \rightarrow \hat{\xi} \text { in } L^{\infty}\left(\Omega, \mathbb{R}^{2}\right) \text { weak * }
$$

From Poincaré inequality (see [3]), there exists a constant $B>0$ such that:

$$
\left\|u_{n}-\int_{\Omega} u_{n}\right\|_{L^{2}(\Omega)} \leq B J\left(u_{n}\right)
$$

Thus from (3.5), we get $\left\|u_{n}-\int_{\Omega} u_{n}\right\|_{L^{2}(\Omega)} \leq B C$. But as $u_{n}+v_{n}=f$, we have:

$$
\int_{\Omega} u_{n}+\underbrace{\int_{\Omega} v_{n}}_{=0 \text { since } v_{n} \in G(\Omega)}=\int_{\Omega} f
$$

Hence $u_{n}$ is bounded in $L^{2}(\Omega)$. And as $u_{n}+v_{n}=f$, we deduce that $v_{n}$ is also bounded in $L^{2}(\Omega)$. Therefore, there exists $\hat{v} \in L^{2}(\Omega)$ such that, up to an extraction,

$$
v_{n} \rightarrow \hat{v} \text { in } L^{2}(\Omega) \text { weak }
$$

Let $\phi \in \mathcal{D}(\Omega)$. We get (using Stokes formula):

$$
\begin{aligned}
\int_{\Omega} v_{n} \phi & =\int_{\Omega} \operatorname{div} \xi_{n} \phi \\
& =-\int_{\Omega} \xi_{n} \nabla \phi+\underbrace{\int_{\partial \Omega} \xi_{n} \cdot N \phi}_{=0 \text { since } v_{n} \in G(\Omega)}
\end{aligned}
$$

$\left(\xi_{n} . N\right.$ is well defined since $\xi_{n} \in L^{2}(\Omega)$ and $\left.\operatorname{div} \xi_{n}=v_{n} \in L^{2}(\Omega)\right)$. Thanks to (3.7):

$$
\int_{\Omega} v_{n} \phi \rightarrow-\int_{\Omega} \hat{\xi} \nabla \phi
$$

But thanks to Stokes formula:

$$
-\int_{\Omega} \hat{\xi} \nabla \phi=\int_{\Omega} \operatorname{div} \hat{\xi} \phi-\underbrace{\int_{\partial \Omega} \hat{\xi} \cdot N \phi}_{=0}
$$

Hence, for all $\phi \in \mathcal{D}(\Omega)$, we have when $n \rightarrow+\infty$ :

$$
\int_{\Omega} v_{n} \phi \rightarrow \int_{\Omega} \operatorname{div} \hat{\xi} \phi
$$

INRIA 
We thus have shown that $v_{n} \rightarrow \operatorname{div} \hat{\xi}$ in $\mathcal{D}^{\prime}(\Omega)$. From (3.10), we deduce that $\hat{v}=\operatorname{div} \hat{\xi}$ in $L^{2}(\Omega)$; and as in the proof of Lemma 2.2 we easily prove that $\xi \cdot N=0$ on $\partial \Omega$. Therefore $\hat{v}$ belongs to $G(\Omega)$. Now up to an extraction, since $J\left(u_{n}\right)$ is bounded and $u_{n}$ is bounded in $L^{2}(\Omega)$, there exists $\hat{u} \in B V(\Omega)$ such that $u_{n} \rightarrow \hat{u}$ in $B V(\Omega)$ weak *.

Let us check what we have shown up to this point:

1. The functional to minimize in (3.1) is convex with respect to its two variables (it is obvious).

2. The infimum in (3.1) is finite (thanks to Lemma 3.1).

3. If $\left(u_{n}, v_{n}\right)$ is a minimizing sequence for (3.1), then there exist $\hat{u} \in B V(\Omega)$ and $\hat{v} \in G(\Omega)$ such that $u_{n} \rightarrow \hat{u} B V$ weak ${ }^{*}$, and $v_{n} \rightarrow \hat{v}$ in $L^{2}(\Omega)$ weak.

To conclude, there remains to prove that $(\hat{u}, \hat{v})$ is a minimizer of $J(u)+\alpha\|v\|_{G(\Omega)}$.

Since $u_{n} \rightarrow \hat{u}$ in $B V(\Omega)$ weak ${ }^{*}$, we know (see [3]) that $\underline{\lim } J\left(u_{n}\right) \geq J(\hat{u})$. And since $\xi_{n}-\hat{\xi}$ in $L^{\infty}\left(\Omega, \mathbb{R}^{2}\right)$ weak $*$, we have $\underline{\lim }\left\|\xi_{n}\right\|_{L^{\infty}\left(\Omega, \mathbb{R}^{2}\right)} \geq\|\hat{\xi}\|_{L^{\infty}\left(\Omega, \mathbb{R}^{2}\right)} \geq\|\hat{v}\|_{G(\Omega)}$ (since $\hat{v}=\operatorname{div} \hat{\xi})$. Hence $\underline{\lim }\left\|v_{n}\right\|_{G(\Omega)} \geq\|\hat{v}\|_{G(\Omega)}$. Therefore:

$$
\underline{\lim }\left(J\left(u_{n}\right)+\alpha\left\|v_{n}\right\|_{G(\Omega)}\right) \geq J(\hat{u})+\alpha\|\hat{v}\|_{G(\Omega)}
$$

which means that $(\hat{u}, \hat{v})$ is a solution of $(3.1)$ (clearly we have $f=u+v$ ).

Remark: The uniqueness of a solution for Meyer problem is an open question.

\section{An algorithm to solve Meyer problem}

In Section 3, we proved the existence of a solution to Meyer problem. We now want to give an algorithm to compute a solution.

\subsection{Total variation minimization as a projection}

Recently, a projection algorithm to solve Rudin-Osher-Fatemi model [23] has been proposed in [9]. Precisely, the problem studied in [9] is:

$$
\inf _{u \in B V(\Omega)}\left(J(u)+\frac{1}{2 \lambda}\|f-u\|_{L^{2}(\Omega)}^{2}\right)
$$

In [9], the following result is shown (in fact, the author in [9] consider the discrete case, but as he mentions, the continuous case is similar): 
Proposition 4.1. The solution of (4.1) is given by:

$$
u=f-P_{G_{\lambda}(\Omega)}(f)
$$

where $P_{G_{\lambda}(\Omega)}$ is the orthogonal projector on $G_{\lambda}(\Omega)$.

Remark: $P_{G_{\lambda}(\Omega)}$ is well defined since $G_{\lambda}(\Omega)$ is a closed convex set of $L^{2}(\Omega)$ (thanks to Lemma 2.2).

\subsection{Solving Meyer problem as a projection}

We now study the algorithm presented in [5] in the continuous setting (whereas in [5], the authors carried out their analysis in a discrete framework).

Let us introduce the following functional defined on $L^{2}(\Omega) \times L^{2}(\Omega)$ :

$$
F_{\lambda, \mu}(u, v)= \begin{cases}J(u)+\frac{1}{2 \lambda}\|f-u-v\|_{L^{2}(\Omega)}^{2} & \text { if }(u, v) \in B V(\Omega) \times G_{\mu}(\Omega) \\ +\infty & \text { otherwise }\end{cases}
$$

We can rewrite $F_{\lambda, \mu}$ in the following way:

$$
F_{\lambda, \mu}(u, v)=J(u)+\frac{1}{2 \lambda}\|f-u-v\|_{L^{2}(\Omega)}^{2}+\chi_{G_{\mu}(\Omega)}(v)
$$

with the convention that $J(u)=+\infty$ if $u \in L^{2}(\Omega) \backslash B V(\Omega)$, and where $\chi_{G_{\mu}(\Omega)}$ is the indicator function of $G_{\mu}(\Omega)$.

The problem we want to solve is:

$$
\inf _{L^{2}(\Omega) \times L^{2}(\Omega)} F_{\lambda, \mu}(u, v)
$$

\subsection{Existence and uniqueness}

We show here that problem (4.5) admits a unique solution.

Proposition 4.2. Let $f \in L^{2}(\Omega)$. There exists a unique couple $(\hat{u}, \hat{v})$ which minimizes $F_{\lambda, \mu}(u, v)$ on $L^{2}(\Omega) \times L^{2}(\Omega)$.

Proof: We split the proof into two steps.

Step 1: Uniqueness

The proof is the same as in [5]. We just put it here for the sake of completeness.

To get the uniqueness, we first remark that $F$ is strictly convex on $B V(\Omega) \times G_{\mu}(\Omega)$, as the sum of a convex function and of a strictly convex function, except in the direction $(u,-u)$ . Hence it suffices to check that if $(\hat{u}, \hat{v})$ is a minimizer of $F$ then for $t \neq 0,(\hat{u}+t \hat{u}, \hat{v}-t \hat{u})$ 
is not a minimizer of $F$. The result is obvious if $\hat{v}-t \hat{u} \in L^{2}(\Omega) \backslash G_{\mu}(\Omega)$. Let us show that if $\hat{v}-t \hat{u} \in G_{\mu}(\Omega)$ then the result is still true. Indeed, if $\hat{v}-t \hat{u} \in G_{\mu}(\Omega)$, we have:

$$
F(\hat{u}+t \hat{u}, \hat{v}-t \hat{u})=F(\hat{u}, \hat{v})+(|1+t|-1) J(\hat{u})
$$

By contradiction, let us assume that there exists $\hat{t} \neq\{-2,0\}$ such that $\hat{v}-\hat{t} \hat{u} \in G_{\mu}(\Omega)$ and

$$
F(\hat{u}+\hat{t} \hat{u}, \hat{v}-\hat{t} \hat{u}) \leq F(\hat{u}, \hat{v})
$$

As $(\hat{u}, \hat{v})$ minimizes $F,(4.7)$ is an equality. From (4.6), we deduce that $(|1+\hat{t}|-1) J(\hat{u})=0$. And as $\hat{t} \neq\{-2,0\}$, we get that $J(\hat{u})=0$. There exists therefore $\gamma \in \mathbb{R}$ such that $\hat{u}=\gamma$ a.e. (we suppose $\Omega$ regular and connected).

1. If $\gamma=0$, then $\hat{u}=0$. Thus $(\hat{u}+\hat{t} \hat{u}, \hat{v}-\hat{t} \hat{u})=(\hat{u}, \hat{v})$.

2. If $\gamma \neq 0$, then $\hat{v}-\hat{t} \hat{u}$ cannot belong to $G_{\mu}(\Omega)$ since its mean is not 0 (see (2.15)). This contradicts our assumption.

There remains to check what happens in the case when $\hat{t}=-2$. In this case, we have: $F(-\hat{u}, \hat{v}+2 \hat{u}) \leq F(\hat{u}, \hat{v})$, i.e. $(-\hat{u}, \hat{v}+2 \hat{u})$ is also a minimizer of $F$. As we assume $\hat{v}+2 \hat{u} \in$ $G_{\mu}(\Omega)$, and as $F$ convex (and as $G_{\mu}(\Omega)$ convex), we get:

$$
F(0, \hat{u}+\hat{v}) \leq \frac{1}{2} F(\hat{u}, \hat{v})+\frac{1}{2} F(-\hat{u}, \hat{v}+2 \hat{u})
$$

And we deduce that $(0, \hat{u}+\hat{v})$ is also a minimizer of $F$. But $F(0, \hat{u}+\hat{v})=F(\hat{u}, \hat{v})$, i.e. $\frac{1}{2 \lambda}\|f-\hat{u}-\hat{v}\|_{L^{2}(\Omega)}^{2}=J(\hat{u})+\frac{1}{2 \lambda}\|f-\hat{u}-\hat{v}\|_{L^{2}(\Omega)}^{2}$. We thus get that $J(\hat{u})=0$, and we conclude as before. Hence there exists at most a unique couple $(\hat{u}, \hat{v}) \in B V(\Omega) \times G_{\mu}(\Omega)$ minimizing $F$ on $L^{2}(\Omega) \times L^{2}(\Omega)$.

Step 2: Existence

Let $\left(u_{n}, v_{n}\right)$ a minimizing sequence for $F_{\lambda, \mu}(u, v)$, i.e.:

$$
F_{\lambda, \mu}\left(u_{n}, v_{n}\right) \rightarrow \inf _{L^{2}(\Omega) \times L^{2}(\Omega)} F_{\lambda, \mu}(u, v)
$$

We easily deduce the three following points:

1.

$$
J\left(u_{n}\right) \leq C
$$

2 .

$$
\left\|f-u_{n}-v_{n}\right\|_{L^{2}(\Omega)}^{2} \leq C
$$

3.

$$
v_{n} \in G_{\mu}
$$


From (4.12), we get that $v_{n}=\operatorname{div} \xi_{n}$, with $\xi_{n}$ such that $\left\|\xi_{n}\right\|_{L^{\infty}\left(\Omega, \mathbb{R}^{2}\right)} \leq \mu$ and $\xi_{n} \cdot N=0$ on $\partial \Omega$.

We also have:

$$
\begin{aligned}
\int_{\Omega} u_{n} v_{n} & =\int_{\Omega} u_{n} \operatorname{div} \xi_{n} \\
& \leq \sup _{\xi \in G_{\mu}(\Omega)} \int_{\Omega} u_{n} \operatorname{div} \xi \\
& \leq \mu \sup _{\xi \in G_{1}(\Omega)} \int_{\Omega} u_{n} \operatorname{div} \xi
\end{aligned}
$$

Hence (using (4.10)):

$$
\int_{\Omega} u_{n} v_{n} \leq \mu J\left(u_{n}\right) \leq C
$$

From (4.11) and (4.13), we deduce that $u_{n}$ and $v_{n}$ are bounded in $L^{2}(\Omega)$. Thanks to (4.10), and since $u_{n}$ and $v_{n}$ are bounded in $L^{2}(\Omega)$, we get that there exists $(\hat{u}, \hat{v})$ such that, up to an extraction:

$$
u_{n} \rightarrow \hat{u} \text { in } B V(\Omega) \text { weak * }
$$

and

$$
v_{n} \rightarrow \hat{v} \text { in } L^{2}(\Omega) \text { weak }
$$

From Lemma 2.2, we know that $\chi_{G_{\mu}(\Omega)}$ is $L^{2}$-weakly lsc, where:

$$
\chi_{G_{\mu}(\Omega)}(v)=\left\{\begin{array}{l}
0 \text { if } v \in G_{\mu}(\Omega) \\
+\infty \text { otherwise }
\end{array}\right.
$$

Since the total variation is lsc for the $B V$ weak * topology, we obtain:

$$
\begin{aligned}
\inf F & =\underline{\lim }\left(J\left(u_{n}\right)+\frac{1}{2 \lambda}\left\|f-u_{n}-v_{n}\right\|_{L^{2}(\Omega)}^{2}+\chi_{G_{\mu}(\Omega)}\left(v_{n}\right)\right) \\
& \geq J(\hat{u})+\frac{1}{2 \lambda}\|f-\hat{u}-\hat{v}\|_{L^{2}(\Omega)}^{2}+\chi_{G_{\mu}(\Omega)}(\hat{v})
\end{aligned}
$$

i.e. $(\hat{u}, \hat{v})$ is a minimizer of $F_{\lambda, \mu}(u, v)$ on $L^{2}(\Omega) \times L^{2}(\Omega)$.

\subsection{Characterization of the minimizer $(\hat{u}, \hat{v})$}

The following result gives a characterization of the minimizer of $F_{\lambda, \mu}$. We will use it in the next subsection to show the convergence of our algorithm.

Proposition 4.3. $(\hat{u}, \hat{v})$ is a minimizer of $F_{\lambda, \mu}(u, v)$ if and only if:

$$
\left\{\begin{array}{c}
\hat{u}=f-\hat{v}-P_{G_{\lambda}(\Omega)}(f-\hat{v}) \\
\hat{v}=P_{G_{\mu}(\Omega)}(f-\hat{u})
\end{array}\right.
$$

INRIA 
Proof: We split the proof into two steps:

Step 1: Necessary condition

Let $(\hat{u}, \hat{v})$ be a minimizer of $F_{\lambda, \mu}(u, v)$. The fact that $\hat{u}=f-\hat{v}-P_{G_{\lambda}(\Omega)}(f-\hat{v})$ comes from Proposition 4.1. The proof of $\hat{v}=P_{G_{\mu}(\Omega)}(f-\hat{u})$ is similar: since $\hat{v}$ is a minimizer of $F(\hat{u},$.$) ,$ we have for all $v$ in $L^{2}(\Omega)$ :

$$
F_{\lambda, \mu}(\hat{u}, \hat{v}) \leq F_{\lambda, \mu}(\hat{u}, v)
$$

which implies.:

$$
0 \in \hat{v}-(f-\hat{u})+\lambda \partial \chi_{G_{\mu}(\Omega)}(\hat{v})
$$

where we denoted by $\partial H$ the subdifferential of $H$ (see $[22,16])$ :

$$
w \in \partial H(u) \Longleftrightarrow H(v) \geq H(u)+(w, v-u)_{L^{2}(\Omega)}, \text { for all } v \text { in } L^{2}(\Omega)
$$

(4.19) means:

$$
f-\hat{u} \in\left(I+\lambda \partial \chi_{G_{\mu}(\Omega)}\right)(\hat{v})
$$

Hence:

$$
\hat{v}=\left(I+\lambda \partial \chi_{G_{\mu}(\Omega)}\right)^{-1}(f-\hat{u})
$$

And it is standard to conclude that:

$$
\hat{v}=P_{G_{\mu}(\Omega)}(f-\hat{u})
$$

Step 2: Sufficient condition

Let $(\hat{u}, \hat{v})$ verifying $(4.17)$. We therefore have:

$$
\left\{\begin{array}{c}
0 \in \hat{u}+\hat{v}-f+\lambda \partial J(\hat{u}) \\
0 \in \hat{u}+\hat{v}-f+\lambda \partial \chi_{G_{\mu}(\Omega)}(\hat{v})
\end{array}\right.
$$

The first line of (4.24) comes from the fact that $\hat{u}=f-\hat{v}-P_{G_{\lambda}(\Omega)}(f-\hat{v})$ (see [9] and [5]), and the second one from the fact that $\hat{v}=P_{G_{\mu}(\Omega)}(f-\hat{u})$. We can rewrite (4.24):

$$
\left\{\begin{array}{c}
\frac{f-\hat{u}-\hat{v}}{\hat{\lambda}} \in \partial J(\hat{u}) \\
\frac{f-\hat{u}}{\lambda} \in \partial \chi_{G_{\mu}(\Omega)}(\hat{v})
\end{array}\right.
$$

which means:

$$
\left\{\begin{aligned}
J(u) & \geq J(\hat{u})+\left(u-\hat{u}, \frac{f-\hat{u}-\hat{v}}{\lambda}\right)_{L^{2}(\Omega) \times L^{2}(\Omega)} \\
\chi_{G_{\mu}(\Omega)}(v) & \geq \chi_{G_{\mu}(\Omega)}(\hat{v})+\left(v-\hat{v}, \frac{f-\hat{u}-\hat{v}}{\lambda}\right)_{L^{2}(\Omega) \times L^{2}(\Omega)}
\end{aligned}\right.
$$

We add the two lines in (4.26), and we obtain:

$$
J(u)+\chi_{G_{\mu}(\Omega)}(v) \geq J(\hat{u})+\chi_{G_{\mu}(\Omega)}(\hat{v})+\left(u-\hat{u}, \frac{f-\hat{u}-\hat{v}}{\lambda}\right)+\left(v-\hat{v}, \frac{f-\hat{u}-\hat{v}}{\lambda}\right)
$$


We then add $\frac{1}{2 \lambda}\|f-u-v\|_{L^{2}(\Omega)}^{2}$ to the two sides of (4.27), and we get:

$$
\begin{aligned}
F_{\lambda, \mu}(u, v) \geq & F_{\lambda, \mu}(\hat{u}, \hat{v})-\frac{1}{2 \lambda}\|f-\hat{u}-\hat{v}\|_{L^{2}(\Omega)}^{2}+\frac{1}{2 \lambda}\|f-u-v\|_{L^{2}(\Omega)}^{2} \\
& +\left(u-\hat{u}, \frac{f-\hat{u}-\hat{v}}{\lambda}\right)+\left(v-\hat{v}, \frac{f-\hat{u}-\hat{v}}{\lambda}\right)
\end{aligned}
$$

To conclude, there remains to show that:

$$
\|f-u-v\|_{L^{2}(\Omega)}^{2}-\|f-\hat{u}-\hat{v}\|_{L^{2}(\Omega)}^{2}+2(u-\hat{u}, f-\hat{u}-\hat{v})+2(v-\hat{v}, f-\hat{u}-\hat{v}) \geq 0
$$

Let us denote by $L(u, v)=\|f-u-v\|_{L^{2}(\Omega)}^{2}$. Since $L$ is convex, we have:

$$
L(u, v) \geq L(\hat{u}, \hat{v})+\left(u-\hat{u}, \frac{\partial L}{\partial u}(\hat{u}, \hat{v})\right)+\left(v-\hat{v}, \frac{\partial L}{\partial v}(\hat{u}, \hat{v})\right)
$$

And (4.29) is just (4.28) which we wanted to show.

The conclusions of Proposition 4.3 naturally lead to the following algorithm for computing the minimizer $(\hat{u}, \hat{v})$. This algorithm was developed and applied in [5].

\section{Algorithm:}

1. Initialization:

$$
u_{0}=v_{0}=0
$$

2. Iterations:

$$
\begin{gathered}
v_{n+1}=P_{G_{\mu}(\Omega)}\left(f-u_{n}\right) \\
u_{n+1}=f-v_{n+1}-P_{G_{\lambda}(\Omega)}\left(f-v_{n+1}\right)
\end{gathered}
$$

3. Stopping test: we stop if

$$
\max \left(\left|u_{n+1}-u_{n}\right|,\left|v_{n+1}-v_{n}\right|\right) \leq \epsilon
$$

Corollary 4.1. The sequence $\left(u_{n}, v_{n}\right)$ (defined by (4.30)-(4.33)) converges to $(\hat{u}, \hat{v}) \in$ $B V(\Omega) \times G_{\mu}(\Omega)$, the unique minimizer of $F_{\lambda, \mu}(u, v)$.

Proof: This a consequence of Proposition 4.1, Proposition 4.3, and the definition of the sequence $\left(u_{n}, v_{n}\right)$ (defined by (4.30)-(4.33)).

INRIA 


\subsection{Study of the limit problem as $\lambda \rightarrow 0$}

We now want to show the link between (3.1) and (4.5). To do so, we consider the following problem

$$
\inf _{(u, v) \in B V(\Omega) \times G(\Omega) / f=u+v} J(u)+\chi_{G_{\mu}}(v)
$$

This is the problem we get when we let $\lambda \rightarrow 0$ in (4.5) (as we will see in Proposition 4.5).

Lemma 4.1. Let $f \in L^{2}(\Omega)$. Let us assume that there exist $\tilde{u} \in B V(\Omega)$ and $\tilde{v} \in G_{\mu}(\Omega)$ such that $f=\tilde{u}+\tilde{v}$. Then there exists $(\hat{u}, \hat{v}) \in B V(\Omega) \times G_{\mu}(\Omega)$ solution of (4.34).

Proof: Using Lemma 2.2, the proof is straightforward.

We now give the link between (3.1) and (4.34).

Proposition 4.4. Let us fix $\alpha>0$ in problem (3.1). Let $(\hat{u}, \hat{v})$ a solution of problem (3.1). We fix $\mu=\|\hat{v}\|_{G}$ in (4.34). Then:

- $(\hat{u}, \hat{v})$ is also a solution of problem (4.34).

- Conversely, any solution $(\bar{u}, \bar{v})$ of (4.34) (with $\mu=\|\hat{v}\|_{G}$ ) is a solution of (3.1). Moreover, we have $\|\bar{v}\|=\mu$ and $J(\bar{u})=J(\hat{u})$.

Proof: The proof is the same as in [5]. We just put it here for the sake of completeness.

We split the proof into two steps.

Step 1:

We first want to show that $(\hat{u}, \hat{v})$ is a solution of (4.34) (with $\left.\mu=\|\hat{v}\|_{G(\Omega)}\right)$. As $(\hat{u}, \hat{v})$ is a solution of (3.1) (the existence of $(\hat{u}, \hat{v})$ is given by Lemma 4.2) and as $\|\hat{v}\|_{G(\Omega)}=\mu$, then $\hat{u}$ is solution of

$$
\inf _{u \in B V(\Omega) / u=f-v, v \in G(\Omega),\|v\|_{G(\Omega)}=\mu} J(u)+\alpha \mu
$$

i.e. $\hat{u}$ is solution of

$$
\inf _{u \in B V(\Omega) / u=f-v, v \in G(\Omega),\|v\|_{G(\Omega)}=\mu} J(u)
$$

Since the set $\left\{u \in B V(\Omega) / u=f-v, v \in G(\Omega),\|v\|_{G(\Omega)}=\mu\right\}$ is contained in $\left\{u \in B V(\Omega) / u=f-v, v \in G(\Omega),\|v\|_{G(\Omega)} \leq \mu\right\}$, we have:

$$
\inf _{u \in B V(\Omega) / u=f-v, v \in G(\Omega),\|v\|_{G(\Omega)}=\mu} J(u) \geq \inf _{u \in B V(\Omega) / u=f-v, v \in G(\Omega),\|v\|_{G(\Omega)} \leq \mu} J(u)
$$

By contradiction, let us assume that

$$
\inf _{u \in B V(\Omega) / u=f-v, v \in G(\Omega),\|v\|_{G(\Omega)}=\mu} J(u)>\inf _{u \in B V(\Omega) / u=f-v, v \in G(\Omega),\|v\|_{G(\Omega)} \leq \mu} J(u)
$$


Thus, there exists $v^{\prime} \in G(\Omega)$ such that $\left\|v^{\prime}\right\|_{G(\Omega)}<\mu$ and

$$
J\left(f-v^{\prime}\right)<\inf _{u \in B V(\Omega) / u=f-v, v \in G(\Omega),\|v\|_{G(\Omega)}=\mu} J(u)
$$

Denoting by $u^{\prime}=f-v^{\prime}$, we have: $J\left(u^{\prime}\right)+\alpha\left\|v^{\prime}\right\|_{G(\Omega)}<J\left(u^{\prime}\right)+\alpha \mu$. But since $(\hat{u}, \hat{v})$ is a solution of $(3.1)$ :

$$
J(\hat{u})+\alpha\|\hat{v}\|_{G(\Omega)} \leq J\left(u^{\prime}\right)+\alpha\left\|v^{\prime}\right\|_{G(\Omega)}<J\left(u^{\prime}\right)+\alpha \mu
$$

Hence (we recall that $\|\hat{v}\|_{G(\Omega)}=\mu$ ), we get from (4.40) that $J(\hat{u})<J\left(u^{\prime}\right)$. This contradicts (4.39). We conclude that (4.38) cannot hold. Hence:

$$
\inf _{u \in B V(\Omega) / u=f-v, v \in G(\Omega),\|v\|_{G(\Omega)}=\mu} J(u)=\inf _{u \in B V(\Omega) / u=f-v, v \in G(\Omega),\|v\|_{G(\Omega)} \leq \mu} J(u)
$$

From (4.36), we see that $\hat{u}$ is a solution of $\inf _{u \in B V(\Omega) / u=f-v, v \in G(\Omega),\|v\|_{G(\Omega)} \leq \mu} J(u)$, i.e. $\hat{u}$ is a solution of

$$
\inf _{u \in B V(\Omega) / u=f-v, v \in G(\Omega)} J(u)+\chi_{G_{\mu}}(v)
$$

Hence $(\hat{u}, \hat{v})$ is also a solution of (4.34).

Step 2:

Let us now consider $(\bar{u}, \bar{v})$ a solution of (4.34) (the existence of $(\bar{u}, \bar{v})$ is given by Lemma 4.1 since $f=\hat{u}+\hat{v}$ with $(\hat{u}, \hat{v}) \in B V(\Omega) \times G(\Omega)$ and $\left.\mu=\|\hat{v}\|_{G(\Omega)}\right)$. We can repeat the computations we made in Step 1 . We get that $\bar{u}$ is a solution of:

$$
\inf _{u \in B V(\Omega) / u=f-v, v \in G(\Omega),\|v\|_{G(\Omega)}=\mu} J(u)+\alpha \mu
$$

We therefore have: $J(\bar{u})+\alpha \mu=J(\hat{u})+\alpha\|\hat{v}\|_{G(\Omega)}$. But as $(\bar{u}, \bar{v})$ is a solution of (4.34), we have $\|\bar{v}\|_{G(\Omega)} \leq \mu$. Hence $J(\bar{u})+\alpha\|\bar{v}\|_{G(\Omega)} \leq J(\hat{u})+\alpha\|\hat{v}\|_{G(\Omega)}$. And since $(\hat{u}, \hat{v})$ is a solution of (3.1), we get that:

$$
J(\bar{u})+\alpha\|\bar{v}\|_{G(\Omega)}=J(\hat{u})+\alpha\|\hat{v}\|_{G(\Omega)}
$$

We thus conclude that $(\bar{u}, \bar{v})$ is a solution of $(3.1)$.

\subsection{Limit when $\lambda \rightarrow 0$}

We precise here the link between (4.5) and (4.34).

Proposition 4.5. Let us fix $\alpha>0$ in (3.1). Let us assume that problem (3.1) has a unique solution $(\hat{u}, \hat{v})$. Set $\mu=\|\hat{v}\|_{G}$ in (4.5) and (4.34). Let us denote by $\left(u_{\lambda}, v_{\lambda}\right)$ the solution of problem (4.5). Then $\left(u_{\lambda}, v_{\lambda}\right)$ converges to $\left(u_{0}, v_{0}\right) \in B V(\Omega) \times G_{\mu}(\Omega)$ as $\lambda$ goes to 0 . Moreover, $\left(u_{0}, v_{0}\right)=(\hat{u}, \hat{v})$ is the solution of problem (4.34). 
Proof: The existence of $(\hat{u}, \hat{v})$ is given by Lemma 3.1. The existence and uniqueness of $\left(u_{\lambda}, v_{\lambda}\right)$ is given by Proposition 4.2. Since $\mu=\|\hat{v}\|_{G}$ in (4.34), we know that problem (4.34) has a solution from Lemma 4.1.

Since $\left(u_{\lambda}, v_{\lambda}\right)$ is the solution of problem (4.5), we have $v_{\lambda} \in G_{\mu}$, and:

$$
F_{\lambda, \mu}\left(u_{\lambda}, v_{\lambda}\right) \leq F_{\lambda, \mu}(\hat{u}, \hat{v})
$$

which means (since $\hat{u}+\hat{v}=f$ )

$$
F_{\lambda, \mu}\left(u_{\lambda}, v_{\lambda}\right) \leq J(\hat{u})
$$

And the left hand-side of (4.46) is given by:

$$
F_{\lambda, \mu}\left(u_{\lambda}, v_{\lambda}\right)=J\left(u_{\lambda}\right)+\frac{1}{2 \lambda}\left\|f-u_{\lambda}-v_{\lambda}\right\|_{L^{2}(\Omega)}^{2}+\chi_{G_{\mu}}\left(v_{\lambda}\right)=J\left(u_{\lambda}\right)+\frac{1}{2 \lambda}\left\|f-u_{\lambda}-v_{\lambda}\right\|_{L^{2}(\Omega)}^{2}
$$

Hence

$$
J\left(u_{\lambda}\right)+\frac{1}{2 \lambda}\left\|f-u_{\lambda}-v_{\lambda}\right\|_{L^{2}(\Omega)}^{2} \leq J(\hat{u})
$$

And

$$
\left\|f-u_{\lambda}-v_{\lambda}\right\|^{2} \leq 2 \lambda J(\hat{u})
$$

We then show as in the proof of Proposition 4.2 that if $\lambda \in[0 ; 1],\left\|u_{\lambda}\right\|_{L^{2}(\Omega)}$ and thus $\left\|v_{\lambda}\right\|_{L^{2}(\Omega)}$ are bounded by a constant $C>0$ which does not depend on $\lambda$.

Consider a sequence $\left(\lambda_{n}\right)$ which goes to 0 as $n \rightarrow+\infty$. Then, up to an extraction, there exists $\left(u_{0}, v_{0}\right) \in B V(\Omega) \times G_{\mu}(\Omega)$ such that $\left(u_{\lambda_{n}}, v_{\lambda_{n}}\right)$ converges to $\left(u_{0}, v_{0}\right)$ in $L^{2}(\Omega)$ weak. By passing to the limit in (4.49), we get: $\left\|f-u_{0}-v_{0}\right\|_{L^{2}(\Omega)}=0$, i.e. $f=u_{0}+v_{0}$.

To conclude the proof of the proposition, there remains to show that $\left(u_{0}, v_{0}\right)$ is a solution of problem (4.34). We first notice that as for all $\lambda \in[0,1],\left\|v_{\lambda}\right\|_{G} \leq \mu$, we get thanks to Lemma 2.2 that $v_{0} \in G_{\mu}$. And from (4.48), we get for all $\lambda>0$ :

$$
J\left(u_{\lambda}\right) \leq J(\hat{u})
$$

Hence $u_{0} \in B V(\Omega)\left(\right.$ since $\left.J\left(u_{0}\right) \leq \underline{\lim } J\left(u_{\lambda}\right) \leq J(\hat{u})\right)$.

Let $(u, v) \in B V(\Omega) \times G_{\mu}(\Omega)$ such that $f=u+v$. We have:

$$
\begin{array}{cc} 
& J(u)+\chi_{G_{\mu}}\left(\frac{v}{\mu}\right)+\frac{1}{2 \lambda} \underbrace{\|f-u-v\|^{2}}_{=0} \\
\geq & J\left(u_{\lambda_{n}}\right)+\chi_{G_{\mu}}\left(\frac{v_{\lambda_{n}}}{\mu}\right)+\frac{1}{2 \lambda_{n}}\left\|f-u_{\lambda_{n}}-v_{\lambda_{n}}\right\|^{2} \\
\geq & J\left(u_{\lambda_{n}}\right)+\chi_{G_{\mu}}\left(\frac{v_{\lambda_{n}}}{\mu}\right)
\end{array}
$$

By passing to the limit as $\lambda \rightarrow 0$, we obtain

$$
J(u)+\chi_{G_{\mu}}\left(\frac{v}{\mu}\right)+\frac{1}{2 \lambda}\|f-u-v\|^{2} \geq J\left(u_{0}\right)+\chi_{G_{\mu}}\left(\frac{v_{0}}{\mu}\right)
$$




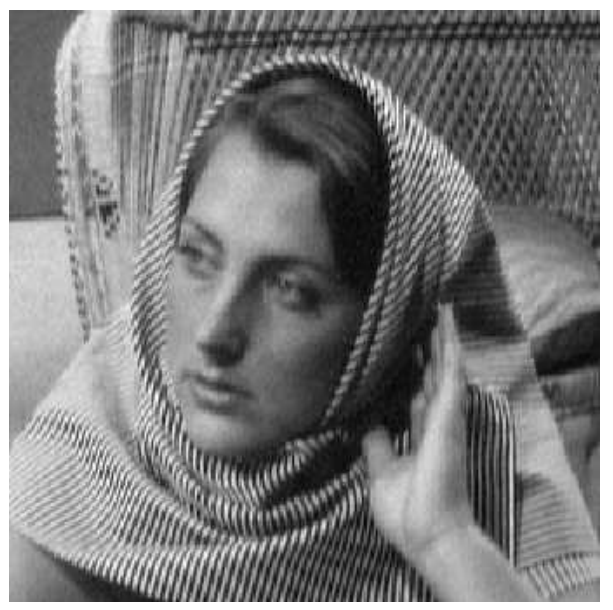

Figure 1: Barbara image

Hence $\left(u_{0}, v_{0}\right)$ is a solution of problem (4.34). And as we have assumed that problem (4.34) has a unique solution, we deduce that $\left(u_{0}, v_{0}\right)=(\hat{u}, \hat{v})$, i.e. $\left(u_{0}, v_{0}\right)$ is the solution of problem (4.34).

\subsection{Numerical results}

We just show here examples of what we obtain with the algorithm (4.30)-(4.33) developed in [5].

We consider the Barbara image (see Figure 1). We first show the decomposition we get for this image on Figure 2: it displays both the $B V$ component and the $G$ component of the original image. Depending on the value we set for $\mu$, the textures present in Figure 1 are separated from the $B V$ component of the image. The larger $\mu$ is, the more the $G$ component contains information. The parameter $\lambda$ controls the $L^{2}$ norm of the residual $f-u-v$. The smaller it is set, the smaller the residual is. To tune the parameters, one first set $\lambda$ to a small value. Then one chooses $\mu$ so that the $G$ component contains the desired amount of information.

On Figure 3, we add a random Gaussian noise of variance $\sigma=30.0$ to the Barbara image, and we then perform the algorithm developed in [5]. As expected by our mathematical analysis, the oscillating patterns of the original Barbara image are put into the $G$ component where their norm is less penalized. More applications of this algorithm are exposed in [5]. 


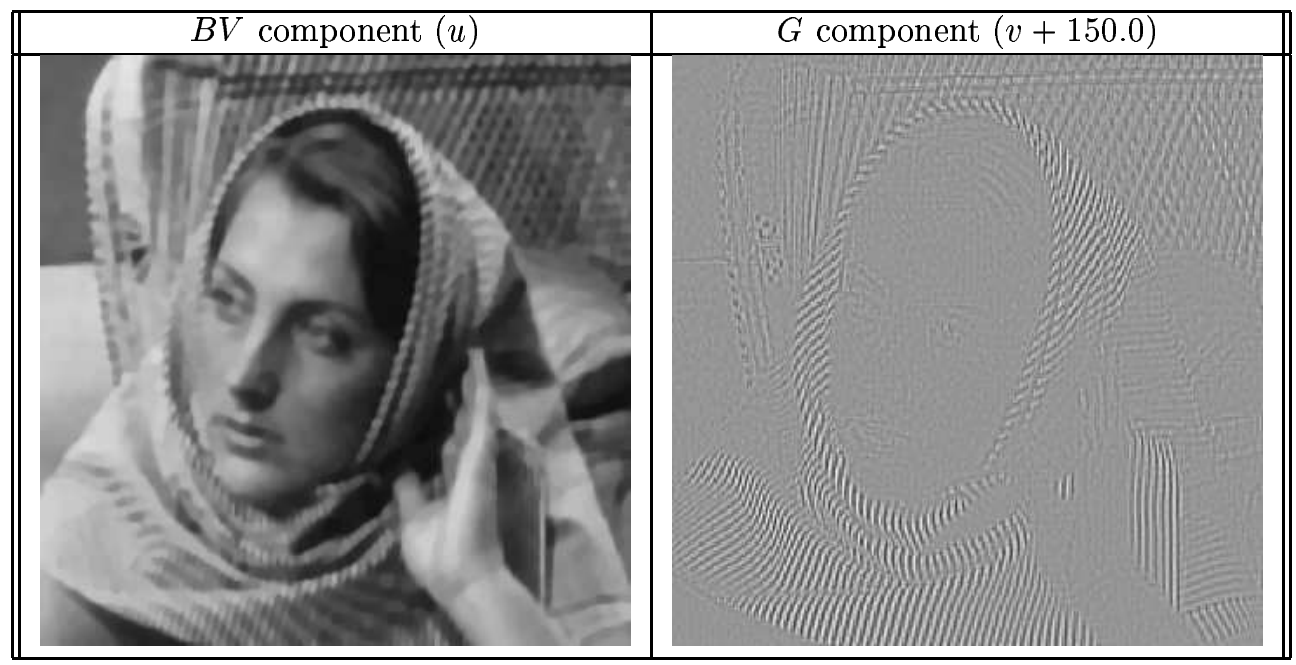

Figure 2: Example of decomposition $(\lambda=0.1, \mu=10)$

\section{References}

[1] R. Adams. Sobolev Spaces. Pure and applied Mathematics. Academic Press, Inc, 1975.

[2] L. Alvarez, Y. Gousseau, and J.M. Morel. Scales in natural images and a consequence on their bounded variation norm. In Scale-Space '99, volume 1682 of Lecture Notes in Computer Science, 1999.

[3] L. Ambrosio, N. Fusco, and D. Pallara. Functions of bounded variations and free discontinuity problems. Oxford mathematical monographs. Oxford University Press, 2000.

[4] G. Aubert and P. Kornprobst. Mathematical Problems in Image Processing, volume 147 of Applied Mathematical Sciences. Springer-Verlag, 2002.

[5] J.F. Aujol, G. Aubert, L. Blanc-Féraud, and A. Chambolle. Decomposing an image: Application to textured images and SAR images, 2003. INRIA Research Report 4704, submitted.

[6] J. Bourgain and H. Brezis. On the equation $\operatorname{div} \mathrm{y}=\mathrm{f}$ and application to control of phases, October 2002. R02022, Laboratoire J.L. Lions.

[7] J. Bourgain and H. Brezis. Sur l' équation div $u=f$. C.R.Math.Acad.Sci.Paris, 334(11):973-976, 2002.

[8] H. Brezis. Analyse fonctionnelle. Théorie et applications. Mathématiques appliquées pour la maitrise. Masson, 1983. 


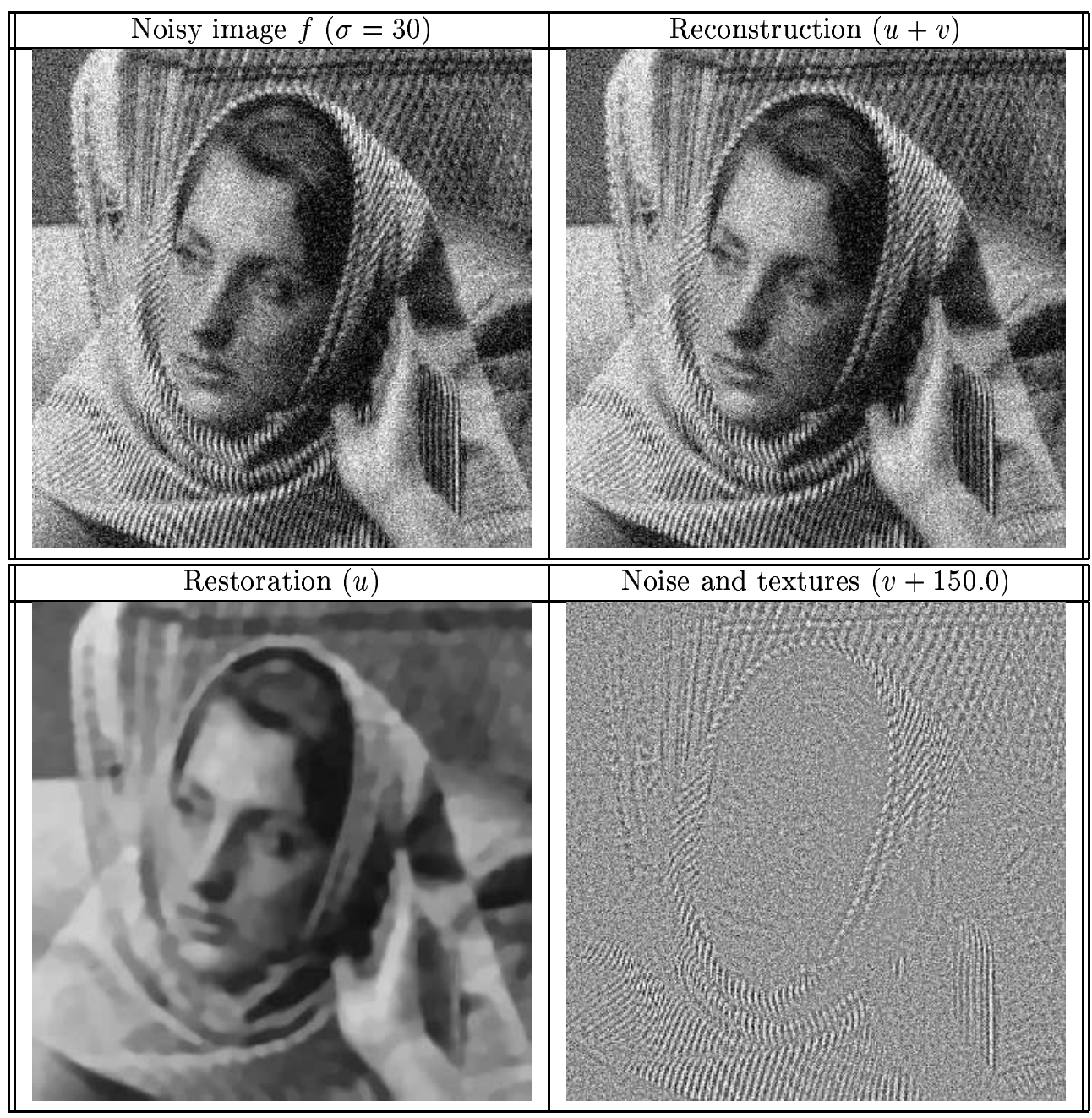

Figure 3: Example of denoising $(\lambda=2.0, \mu=120)$

INRIA 
[9] A. Chambolle. An algorithm for total variation minimization and applications, 2003. To appear in JMIV.

[10] A. Chambolle and P.L. Lions. Image recovery via total variation minimization and related problems. Numerische Mathematik, 76(3):167-188, 1997.

[11] R. Dautray and J.L. Lions. Analyse mathématique et calcul numérique pour les sciences et les techniques, volume 4. Masson, 1988.

[12] L.C. Evans. Partial Differential Equations, volume 19 of Graduate Studies in Mathematics. American Mathematical Society, 1991.

[13] L.C. Evans and R.F. Gariepy. Mesure theory and fine properties of functions. Studies in advanced Mathematics. CRC Press, 1992.

[14] D. Gilbarg and N.S. Trudinger. Elliptic Partial Differential Equations of Second Order, volume 28 of Princeton Mathematical Series. Springer-Verlag, 1970.

[15] P. Grisvard. Elliptic problems in nonsmooth domains, volume 24 of Monographs and studies in Mathematics. Pitman, 1985.

[16] J.B. Hiriart-Urruty and C. Lemarechal. Convex Analysis ans Minimisation Algorithms I, volume 305 of Grundlehren der mathematischen Wissenschaften. Springer-Verlag, 1993.

[17] J.L. Lions and E. Magenes. Problèmes aux limites non homogènes, volume 1. Dunod, 1968.

[18] Yves Meyer. Oscillating patterns in image processing and in some nonlinear evolution equations, March 2001. The Fifteenth Dean Jacquelines B. Lewis Memorial Lectures.

[19] A.A. Miljutin. A priori estimates for solutions of second order linear elliptic equations. Mat. Sbornik, pages 459-474, 1960. Russian.

[20] C. Miranda. Partial differential equations of elliptic type. Springer-Verlag, second edition, 1970.

[21] S.J. Osher, A. Sole, and L.A. Vese. Image decomposition and restoration using total variation minimization and the $\mathrm{H}^{-1}$ norm, October 2002. UCLA C.A.M. Report 02-57.

[22] T. Rockafellar. Convex Analysis, volume 224 of Grundlehren der mathematischen Wissenschaften. Princeton University Press, second edition, 1983.

[23] L. Rudin, S. Osher, and E. Fatemi. Nonlinear total variation based noise removal algorithms. Physica D, 60:259-268, 1992.

[24] R. Temam. Navier Stokes equations. Elsevier Science Publishers B.V., 1984. 
[25] L.A. Vese and S.J. Osher. Modeling textures with total variation minimization and oscillating patterns in image processing, May 2002. UCLA C.A.M. Report 02-19. To appear in Journal of Scientific Computing. 


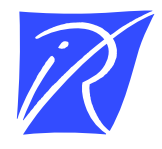

\section{Unité de recherche INRIA Sophia Antipolis 2004, route des Lucioles - BP 93 - 06902 Sophia Antipolis Cedex (France)}

Unité de recherche INRIA Futurs : Parc Club Orsay Université - ZAC des Vignes 4, rue Jacques Monod - 91893 ORSAY Cedex (France)

Unité de recherche INRIA Lorraine : LORIA, Technopôle de Nancy-Brabois - Campus scientifique 615, rue du Jardin Botanique - BP 101 - 54602 Villers-lès-Nancy Cedex (France)

Unité de recherche INRIA Rennes : IRISA, Campus universitaire de Beaulieu - 35042 Rennes Cedex (France)

Unité de recherche INRIA Rhône-Alpes : 655, avenue de l'Europe - 38334 Montbonnot Saint-Ismier (France)

Unité de recherche INRIA Rocquencourt : Domaine de Voluceau - Rocquencourt - BP 105 - 78153 Le Chesnay Cedex (France)

INRIA - Domaine de Voluceau - Rocquencourt, BP 105 - 78153 Le Chesnay Cedex (France)

http://www.inria.fr

ISSN 0249-6399 